\title{
Individualized perfusion targets in hypoxic ischemic brain injury after cardiac arrest
}

\author{
Mypinder S. Sekhon ${ }^{1,4^{*}}$ and Donald E. Griesdale $e^{1,2,3}$
}

\begin{abstract}
Secondary injury is a major determinant of outcome in hypoxic ischemic brain injury (HIBI) after cardiac arrest and may be mitigated by optimizing cerebral oxygen delivery $\left(\mathrm{CDO}_{2}\right) \cdot \mathrm{CDO}_{2}$ is determined by cerebral blood flow (CBF), which is dependent upon mean arterial pressure (MAP). In health, CBF remains constant over the MAP range through cerebral autoregulation. In $\mathrm{HIBI}$, the zone of intact cerebral autoregulation is narrowed and varies for each patient. Maintaining MAP within the intact autoregulation zone may mitigate ischemia, hyperemia and secondary injury. The optimal MAP in individual patients can be determined using real time autoregulation monitoring techniques.
\end{abstract}

Keywords: Hypoxemic ischemic brain injury, Cardiac arrest, Cerebral autoregulation, Mean arterial pressure, Cerebral oxygen delivery, Secondary injury

\section{Introduction}

Cardiac arrest leads to immediate cessation of cerebral blood flow (CBF) and secondary injury after return of spontaneous circulation (ROSC) [1]. This cerebral insult, termed hypoxic ischemic brain injury (HIBI), is the primary determinant of outcome [2]. Despite timely ROSC, ongoing cerebrovascular dysfunction can lead to secondary injury and worse neurologic outcome. HIBI management focuses on mitigating this secondary injury by optimizing blood glucose, temperature and, importantly, the balance between cerebral oxygen delivery $\left(\mathrm{CDO}_{2}\right)$ and utilization [3].

$\mathrm{CDO}_{2}$ is dependent upon CBF, which in turn is principally determined by mean arterial pressure (MAP). Recognizing the lack of high quality data, the American Heart Association recommends maintaining a MAP $\geq 65 \mathrm{mmHg}$ and systolic blood pressure $\geq 90 \mathrm{mmHg}$ after ROSC [4]. These uniform hemodynamic thresholds fail to account for individual variability within patient and disease pathophysiology. Ultimately, this "one-size fits all" approach may subject HIBI patients to secondary ischemic injury and worse outcome [5].

\footnotetext{
* Correspondence: mypindersekhon@gmail.com

${ }^{1}$ Department of Medicine, Division of Critical Care Medicine, Vancouver General Hospital, West 12th Avenue, University of British Columbia, Vancouver, BC V5Z 1M9, Canada

${ }^{4}$ Critical Care Medicine, Vancouver General Hospital, Room 2438, Jim Pattison Pavilion, 2nd Floor, 855 West 12th Avenue, Vancouver, BC V5Z 1M9, Canada Full list of author information is available at the end of the article
}

Cerebral autoregulation, through compensatory vasodilation and vasoconstriction, is the innate ability of the cerebrovasculature to maintain stable CBF over a wide range of MAP [6]. In health, autoregulation ensures the cerebral parenchyma is protected from both ischemia and hyperemia. At the cellular level, endothelial release of vasodilators and vasoconstrictors as well as activation of L-type calcium channels with signaling from adjoining astrocytes are responsible for the observed CBF homeostasis in response to fluctuations in arteriole blood pressure [7]. Historically, complete loss of autoregulation was thought to occur after brain injury, resulting in a linear relationship between CBF and MAP [8]. In fact, autoregulation may remain intact, albeit with a narrowed and rightshifted intact zone after HIBI [9]. Importantly, the specific zone of intact autoregulation appears to have significant heterogeneity between individual patients after HIBI [9, 10]. Our ability to detect the precise MAP threshold at which autoregulation occurs may eventually lead to personalized perfusion targets after cardiac arrest. With this in mind, the purpose of this viewpoint is to discuss the advances in autoregulation monitoring in patients with HIBI. Furthermore, we discuss how this technology may dramatically shift our thinking from the traditional fixed MAP thresholds to personalized perfusion targets. 


\section{Methods}

In this viewpoint, we conducted a non-systematic literature search on MEDLINE and EMBASE to identify pertinent citations which were referenced in our study. We did not examine conference abstract proceedings. Subsequently, study abstracts were reviewed and manuscripts describing autoregulation monitoring techniques after cardiac arrest were used as references.

\section{Discussion}

The acute post resuscitative phase of HIBI is characterized by a "no reflow" phenomenon resulting in persistent cerebral oligemia despite restoration of cardiac output. No reflow, in part, stems from diffuse cerebrovascular inflammation, intravascular microthrombi [11], dysfunctional nitric oxide signaling and perivascular cerebral edema culminating in increased cerebrovascular resistance and decreased CBF [12]. Hypotension, particularly below the lower limit of autoregulation, during this critical period may further exacerbate ongoing cerebral ischemia and secondary injury [13]. Trzeciak and colleagues retrospectively demonstrated an association between hypotension (systolic blood pressure $<90 \mathrm{mmHg}$ ) and increased in-hospital death (OR 2.7 [2.5-3.0]) in 8736 cardiac arrest patients [14]. Furthermore, Laurikkala and colleagues prospectively found an association between unfavorable neurological outcome and time spent below a MAP of $70 \mathrm{mmHg}$ in the first $48 \mathrm{~h}$ after ROSC [15]. Multiple additional observational studies demonstrate associations between hypotension after ROSC and poor neurologic outcome and death [14, 16-21]. Our research group performed a systematic review and concluded that improved neurologic outcomes after cardiac arrest are associated with increased blood pressure, albeit with significant between-study heterogeneity [5].

Although uniformly increased MAP targets seem intuitive to mitigate secondary injury in HIBI and are supported by emerging observational literature, guideline statements recommend a MAP threshold of $\geq 65 \mathrm{mmHg}$ [4]. The persistence of this universal MAP threshold in guidelines is in part driven by the lack of large randomized control trial data. Recently authors have argued to move beyond the question of uniformly increased MAP versus current guideline-based MAP targets and instead focus on individualized hemodynamic thresholds in the setting of post-cardiac arrest patient physiology and preexisting comorbidities [22, 23].

Cerebral autoregulation monitoring allows us to determine individualized MAP thresholds, thereby moving towards a personalized resuscitative strategy. Sundgreen and colleagues conducted a sentinel prospective physiologic study to assess autoregulation in patients after cardiac arrest using transcranial Doppler to assess middle cerebral artery blood flow velocity while incrementally augmenting MAP with intravenous norepinephrine [9]. There was considerable between-patient variability in the zone of intact autoregulation. In a third of patients, the zone of autoregulation was markedly narrowed and right shifted above a MAP of $100 \mathrm{mmHg}$ [9]. In the remaining patients, nearly half demonstrated a complete loss of autoregulation, highlighting the marked heterogeneity in cerebrovascular hemodynamics between HIBI patients [9]. Although transcranial Doppler-based autoregulation assessment is well established, inter-observer reliability, adequate middle cerebral artery insonation windows and the inability to perform long-term continuous monitoring limit its widespread use. Therefore, alternative methods of autoregulation monitoring are required.

Cerebral autoregulation can also be assessed noninvasively using the relationship between MAP and regional saturation of oxygen $\left(\mathrm{rSO}_{2}\right)$ using near infrared spectroscopy (NIRS) [24]. NIRS emits infrared light at frequencies between 700 and $950 \mathrm{~mm}$ where is it subsequently refracted or absorbed in the first 1 to $2 \mathrm{~cm}$ of frontal lobe tissue and estimates a percentage of a ratio of oxygenated versus deoxygenated hemoglobin, termed the $\left(\mathrm{rSO}_{2}\right)$ [25]. To assess autoregulation, we can observe how fluctuations in MAP will result in changes to $\mathrm{rSO}_{2}$. When autoregulation is compromised, $\mathrm{rSO}_{2}$ is positively correlated with MAP (e.g., increasing MAP leads to increasing $\mathrm{rSO}_{2}$ ) [24]. Conversely, when autoregulation is intact, $\mathrm{rSO}_{2}$ remains constant despite fluctuations in MAP [24]. Over time, a moving correlation coefficient between MAP and $\mathrm{rSO}_{2}$ can be calculated, termed COx. A positive COx indicates dysfunctional autoregulation [26] and a negative or near zero COx indicates intact autoregulation [26]. Generally, autoregulation is considered to be preserved when the COx is less than 0.3 [27]. After plotting COx (y axis) versus the individual patient's MAP range, a $U$ shaped curve can be produced and the nadir of this curve corresponds to the optimal MAP for each individual patient (Fig. 1) [28]. (Figure 1 is original for this manuscript and was used with approval from the University of British Columbia clinical research ethics board-H15-01606.)

COx-based autoregulation monitoring following cardiac arrest is well studied in pediatric swine models of neonatal HIBI. As part of these animal studies, Brady et al. established that COx was able to accurately detect the lower limit of autoregulation with a sensitivity of $92 \%$ and receiver operator characteristics area under the curve of 0.89 . Furthermore, these authors also demonstrated that the identification of the lower limit of autoregulation using $\mathrm{COx}$ was in agreement compared to invasive monitoring techniques using laser Doppler flow and pressure reactivity index [29].

Clinically, Howlett et al. [30] have shown that neonates who spent a greater proportion of time below the optimal MAP had an increased burden of basal ganglia, white matter and thalamus injuries after HIBI. Similarly, 


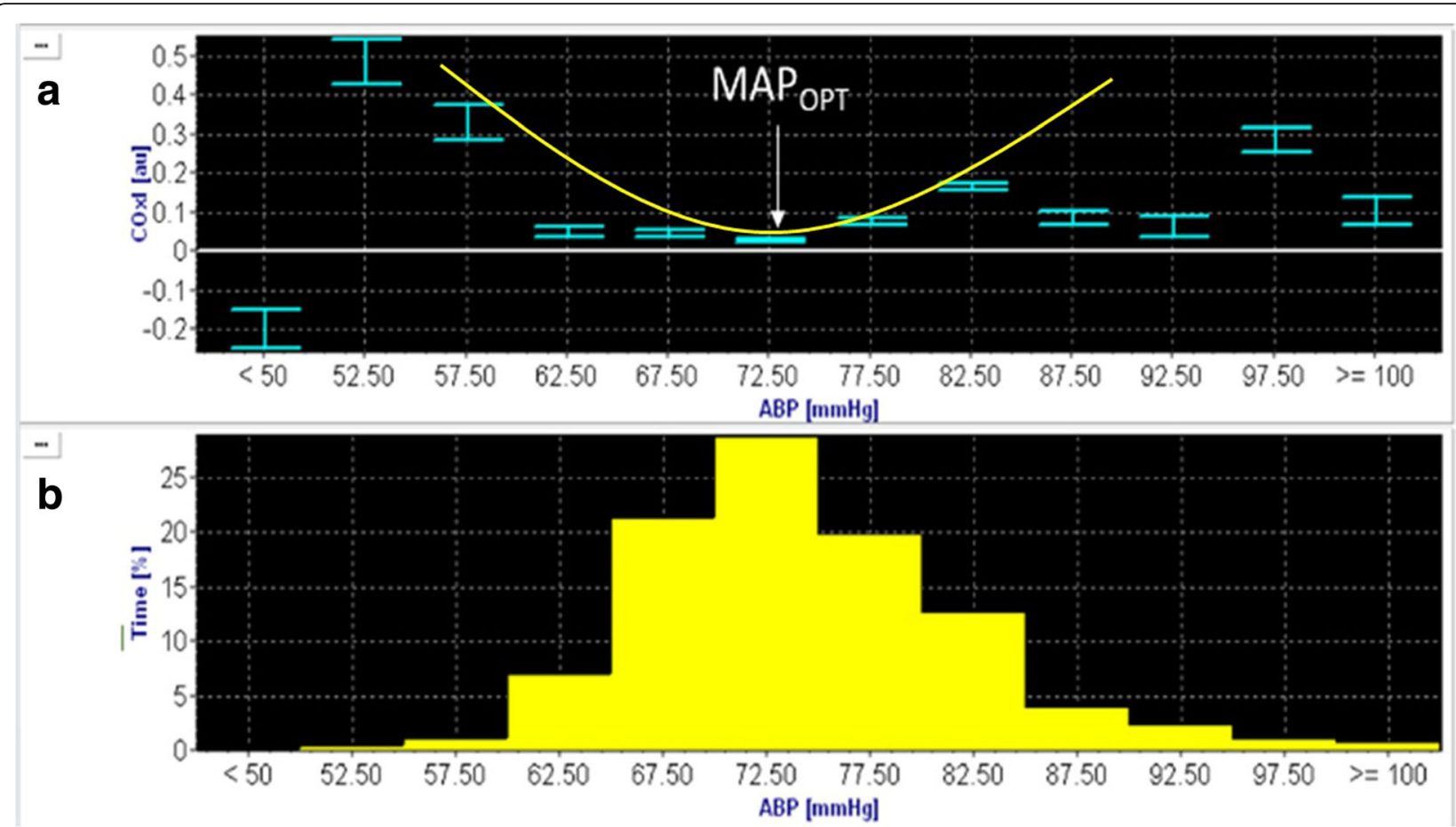

Fig. 1 Autoregulation-based identification of optimal mean arterial pressure after hypoxic ischemic brain injury. a A best fit U-shaped curve with the nadir of the curve representing the optimal mean arterial pressure (MAP OPT). The COx is plotted along the $y$-axis and mean arterial pressure in 5-mmHg bins on the $\mathrm{x}$-axis, denoted as $A B P$. $\mathbf{b}$ The amount of time during the monitoring period spent within each $5-\mathrm{mmHg}$ bin of mean arterial pressure. The total duration of time is denoted as a percentage on the $y$-axis with each $5-\mathrm{mmHg}$ mean arterial pressure bin on the $\mathrm{x}$-axis. This figure is original for this manuscript

Lee and colleagues have shown that deviation from the identified optimal MAP using COx was associated with magnetic resonance imaging features of HIBI in neonates after perinatal asphyxia [31]. These authors have also shown that deviation from optimal MAP was associated with poor neurologic outcome in 36 pediatric cardiac arrest patients [32]. Burton et al. [33] confirmed these results by studying 28 neonates with HIBI after asphyxia and found worse motor and cognitive function in those patients who had a greater time of deviation below the identified optimal MAP with NIRS-based autoregulation assessment.

In adult cardiac arrest subjects, Ameloot and colleagues retrospectively calculated $\mathrm{COx}$ and demonstrated that cerebral autoregulation was present in 33 of 51 patients [10]. Pham and colleagues observed that COx was significantly higher when comparing non-survivors to survivors in patients after cardiac arrest [34]. Interestingly, there was no relationship between the absolute value of $\mathrm{rSO}_{2}$ and outcome in this cohort. Our research group recently conducted a prospective observational study of real-time cerebral autoregulation monitoring using $\mathrm{COx}$ in 20 patients with HIBI following cardiac arrest [28]. Importantly, we were also able to demonstrate that this technique is feasible as we generated U-shaped autoregulation curves and identified the optimal MAP in 19 of 20 patients. The mean optimal MAP (MAP ${ }_{\text {OPT }}$ ) was $75 \mathrm{mmHg}$ (standard deviation (SD) 10). The mean percentage of time spent outside $5 \mathrm{mmHg}$ from $\mathrm{MAP}_{\mathrm{OPT}}$ was $47 \%$ (SD 21\%). Dysfunctional autoregulation (percentage of time with $\mathrm{COx}$ at 0.3 or above) occurred in $11 \%$ (SD 17\%) of measurement time [28]. Furthermore, we demonstrated an association with increased $\mathrm{COx}$ and hyperthermia, highlighting the importance of temperature regulation in cerebrovascular reactivity after HIBI [28]. These findings are consistent with dysfunctional cerebral autoregulation observed during hyperthermia $\left(>38^{\circ} \mathrm{C}\right)$ in patients with traumatic brain injury [35]. Although hyperthermia appears to be associated with dysfunctional cerebral autoregulation after brain injury, currently no definitive data suggest a difference in the preservation of autoregulation in patients managed with normothermia $\left(36{ }^{\circ} \mathrm{C}\right)$ versus hypothermia $\left(33^{\circ} \mathrm{C}\right)$, making it a logical question for future research.

Significant work remains to delineate the role of COxbased autoregulation monitoring in adult HIBI patients. In traumatic brain injury, a similar Pearson correlation coefficient between MAP and intracranial pressure (PRx) can individualize cerebral perfusion pressure thresholds [36]. Investigators have demonstrated that perfusion within the intact zone of autoregulation as determined by $\mathrm{PRx}$ is 
associated with improved cerebral oxygenation [37]. Furthermore, a large observational outcome study has suggested that perfusion within $5 \mathrm{mmHg}$ of the optimal cerebral perfusion pressure after traumatic brain injury is associated with improved long-term neurological outcomes [38]. With this in mind, further examining the relationship of individualized cerebral autoregulation thresholds and neurologic outcomes in patients with HIBI is a logical next step. Additionally, the changes that occur with autoregulation in adult HIBI patients and key physiologic modulators, including arterial carbon dioxide concentration, core body temperature, intravenous sedatives and vasoactive agents, are essential research questions. Finally, a comparison of the identification of optimal MAP using COx versus direct measures of $\mathrm{CBF}$ in an adult model of HIBI is imperative moving forward.

Although using cerebral autoregulation monitoring to titrate MAP is an intriguing therapeutic strategy, several limitations need to be addressed. Firstly, cardiac arrest often results in impaired left ventricular function, which may variably tolerate the increase in afterload from exogenous vasopressor administration for targeting right-shifted optimal MAP using COx. Furthermore, in patients who suffer a primary cardiac arrest, administration of vasopressors to increased MAP will increase ventricular wall stress and myocardial oxygen consumption and potentially extend the infarction core. High doses of vasopressors themselves have significant risks, including arrhythmias, increased pulmonary vascular permeability and myocardial, mesenteric and digital ischemia. Therefore, the risks associated with increased MAP targets must be weighed against the potential benefits of improved cerebral perfusion and oxygenation.

NIRS monitoring itself has significant limitations, notably the contamination of the $\mathrm{rSO}_{2}$ signal from both ambient light and extracranial blood flow, albeit, the precise proportion of signal interference from scalp blood flow is not well established [39]. Furthermore, skin moisture stemming from forehead perspiration can lead to inadequate adherence of NIRS probes and inaccurate signal [39]. It should be recognized that NIRS only samples the 1 to $2 \mathrm{~cm}$ of superficial frontal lobe tissue beneath the forehead and, as such, may not reflect regional differences in autoregulation in crucial cerebral structures particularly susceptible to injury after HIBI, including the grey-white matter interface, basal ganglia and hippocampi [39]. Finally, important physiologic variables such as acute fluctuations in hemoglobin concentration, arterial carbon dioxide and oxygen tension can influence the NIRS signal, thereby changing the $\mathrm{rSO}_{2}$ independently of fluctuations in MAP. This is a major pitfall in NIRS-based autoregulation monitoring in that the relationship between MAP and $\mathrm{rSO}_{2}$, which generates $\mathrm{COx}$, can be altered by acute changes in these other physiologic variables.

\section{Conclusions}

Autoregulation monitoring represents a recent advance and unique opportunity to further develop our understanding of the perturbations in the cerebrovascular hemodynamics in patients with HIBI. Cerebral autoregulation monitoring provides an opportunity to individualize perfusion targets rather than the traditional "one size fits all" approach.

\begin{abstract}
Abbreviations
CA: Cardiac arrest; CBF: Cerebral blood flow; $\mathrm{CDO}_{2}$ : Cerebral oxygen delivery; $\mathrm{HIBI}$ : Hypoxic ischemic brain injury; ROSC: Return of spontaneous circulation; $\mathrm{rSO}_{2}$ : Regional saturation of oxygen
\end{abstract}

\section{Acknowledgements}

Not applicable.

\section{Funding}

Dr. Sekhon is funded by the Vancouver Coastal Health Research Institute. Dr. Griesdale is funded by the VGH \& UBC Hospital Foundation Best of Health Fund.

Availability of data and materials

Not applicable.

Authors' contributions

MS is the primary author and contributed the majority of the manuscript preparation and background research. DG contributed to manuscript preparation. All authors read and approved the final manuscript.

\section{Authors' information}

Not applicable.

Ethics approval and consent to participate

Approval was obtained from the University of British Columbia clinical research ethics board (H15-01606).

Consent for publication

Not applicable.

\section{Competing interests}

None to report.

\section{Publisher's Note}

Springer Nature remains neutral with regard to jurisdictional claims in published maps and institutional affiliations.

\section{Author details}

'Department of Medicine, Division of Critical Care Medicine, Vancouver General Hospital, West 12th Avenue, University of British Columbia, Vancouver, BC V5Z 1M9, Canada. ${ }^{2}$ Department of Anesthesiology, Pharmacology and Therapeutics, Vancouver General Hospital, West 12th Avenue, University of British Columbia, Vancouver, BC V5Z 1M9, Canada. ${ }^{3}$ Centre for Clinical Epidemiology and Evaluation, Vancouver Coastal Health Research Institute, 899 West 12th Avenue, University of British Columbia, Vancouver, BC V5Z 1M9, Canada. ${ }^{4}$ Critical Care Medicine, Vancouver General Hospital, Room 2438, Jim Pattison Pavilion, 2nd Floor, 855 West 12th Avenue, Vancouver, BC V5Z 1M9, Canada.

Published online: 24 October 2017

\section{References}

1. Sekhon MS, Ainslie PN, Griesdale DE. Clinical pathophysiology of hypoxic ischemic brain injury after cardiac arrest: a "two-hit" model. Crit Care. 2017; 21:90.

2. Nielsen N, Wetterslev J, Cronberg T, Erlinge D, Gasche $Y$, Hassager $C$, et al. Targeted temperature management at $33{ }^{\circ} \mathrm{C}$ versus $36{ }^{\circ} \mathrm{C}$ after cardiac arrest. N Engl J Med. 2013;369:2197-206. 
3. Nolan JP, Neumar RW, Adrie C, Aibiki M, Berg RA, Böttiger BW, et al. Postcardiac arrest syndrome: epidemiology, pathophysiology, treatment, and prognostication. A Scientific Statement from the International Liaison Committee on Resuscitation; the American Heart Association Emergency Cardiovascular Care Committee; the Coun. Resuscitation. 2008;79:350-79.

4. Peberdy MA, Callaway CW, Neumar RW, Geocadin RG, Zimmerman JL, Donnino M, et al. Part 9: Post-cardiac arrest care: 2010 American Heart Association guidelines for cardiopulmonary resuscitation and emergency cardiovascular care. Circulation. 2010;122:S768-86.

5. Bhate TD, McDonald B, Sekhon MS, Griesdale DEG. Association between blood pressure and outcomes in patients after cardiac arrest: a systematic review. Resuscitation. 2015;97:1-6.

6. Brady K, Joshi B, Zweifel C, Smielewski P, Czosnyka M, Easley RB, et al. Realtime continuous monitoring of cerebral blood flow autoregulation using near-infrared spectroscopy in patients undergoing cardiopulmonary bypass. Stroke. 2010;41:1951-6.

7. Filosa JA, Iddings JA. Astrocyte regulation of cerebral vascular tone. AJP Hear Circ Physiol. 2013;305:H609-19.

8. Nishizawa $\mathrm{H}$, Kudoh I. Cerebral autoregulation is impaired in patients resuscitated after cardiac arrest. Acta Anaesthesiol Scand. 1996;40:1149-53.

9. Sundgreen C, Larsen FS, Herzog TM, Knudsen GM, Boesgaard S, Aldershvile J. Autoregulation of cerebral blood flow in patients resuscitated from cardiac arrest. Stroke. 2001;32:128-32.

10. Ameloot K, Genbrugge C, Meex I, Jans F, Boer W, Vander Laenen M, et al. An observational near-infrared spectroscopy study on cerebral autoregulation in post-cardiac arrest patients: time to drop "one-size-fits-all" hemodynamic targets? Resuscitation. 2015;90:121-6.

11. Busl KM, Greer DM. Hypoxic-ischemic brain injury: pathophysiology, neuropathology and mechanisms. NeuroRehabilitation. 2010;26:5-13.

12. Böttiger BW, Krumnikl JJ, Gass P, Schmitz B, Motsch J, Martin E. The cerebral "no-reflow" phenomenon after cardiac arrest in rats-influence of low-flow reperfusion. Resuscitation. 1997;34:79-87.

13. Lee JK, Brady KM, Mytar JO, Kibler KK, Carter EL, Hirsch KG, et al. Cerebral blood flow and cerebrovascular autoregulation in a swine model of pediatric cardiac arrest and hypothermia. Crit Care Med. 2011;39:2337-45.

14. Trzeciak S, Jones AE, Kilgannon JH, Milcarek B, Hunter K, Shapiro NI, et al. Significance of arterial hypotension after resuscitation from cardiac arrest. Crit Care Med. 2009;37:2895-903.

15. Laurikkala J, Wilkman E, Pettilä V, Kurola J, Reinikainen M, Hoppu S, et al. Mean arterial pressure and vasopressor load after out-of-hospital cardiac arrest: associations with one-year neurologic outcome. Resuscitation. 2016;105:116-22.

16. Ameloot K, Meex I, Genbrugge C, Jans F, Boer W, Verhaert D, et al. Hemodynamic targets during therapeutic hypothermia after cardiac arrest: a prospective observational study. Resuscitation. 2015:91:56-62.

17. Young MN, Hollenbeck RD, Pollock JS, Giuseffi JL, Wang L, Harrell FE, et al. Higher achieved mean arterial pressure during therapeutic hypothermia is not associated with neurologically intact survival following cardiac arrest. Resuscitation. 2015;88:158-64.

18. Kilgannon JH, Roberts BW, Jones AE, Mittal N, Cohen E, Mitchell J, et al. Arterial blood pressure and neurologic outcome after resuscitation from cardiac arrest. Crit Care Med. 2014;42:2083-91.

19. Bray JE, Bernard S, Cantwell K, Stephenson M, Smith K, VACAR Steering Committee. The association between systolic blood pressure on arrival at hospital and outcome in adults surviving from out-of-hospital cardiac arrests of presumed cardiac aetiology. Resuscitation. 2014;85:509-15.

20. Kaji AH, Hanif AM, Thomas JL, Niemann JT. Out-of-hospital cardiac arrest: Early in-hospital hypotension versus out-of-hospital factors in predicting inhospital mortality among those surviving to hospital admission. Resuscitation. 2011:82:1314-7.

21. Russo JJ, James TE, Hibbert B, Yousef A, Osborne C, Wells GA, et al. Impact of mean arterial pressure on clinical outcomes in comatose survivors of out-ofhospital cardiac arrest: insights from the University of Ottawa Heart Institute Regional Cardiac Arrest Registry (CAPITAL-CARe). Resuscitation. 2017;113:27-32.

22. Soar J, Callaway CW, Aibiki M, Böttiger BW, Brooks SC, Deakin CD, et al. Part 4: Advanced life support. Resuscitation. 2015;95:e71-120.

23. Gaieski DF, Beylin ME, Abella BS, Grossestreuer AV, Perman SM. What is the optimal post-arrest hemodynamic strategy? Towards personalized resuscitation strategies. Intensive Care Med. 2014;40:466.

24. Zweifel C, Castellani G, Czosnyka M, Carrera E, Brady KM, Kirkpatrick PJ, et al. Continuous assessment of cerebral autoregulation with near-infrared spectroscopy in adults after subarachnoid hemorrhage. Stroke. 2010;41:1963-8.
25. Brady KM, Mytar JO, Kibler KK, Hogue CW, Lee JK, Czosnyka M, et al. Noninvasive autoregulation monitoring with and without intracranial pressure in the naive piglet brain. Anesth Analg. 2010;111:191-5.

26. Brady KM, Lee JK, Kibler KK, Smielewski P, Czosnyka M, Easley RB, et al. Continuous time-domain analysis of cerebrovascular autoregulation using near-infrared spectroscopy. Stroke. 2007;38:2818-25.

27. Lee JK, Kibler KK, Benni PB, Easley RB, Czosnyka M, Smielewski P, et al. Cerebrovascular reactivity measured by near-infrared spectroscopy. Stroke. 2009:40:1820-6.

28. Sekhon MS, Smielewski P, Bhate TD, Brasher PM, Foster D, Menon DK, et al. Using the relationship between brain tissue regional saturation of oxygen and mean arterial pressure to determine the optimal mean arterial pressure in patients following cardiac arrest: a pilot proof-of-concept study. Resuscitation. 2016;106:120-5.

29. Brady KM, Lee JK, Kibler KK, Easley RB, Koehler RC, Shaffner DH. Continuous measurement of autoregulation by spontaneous fluctuations in cerebral perfusion pressure: comparison of 3 methods. Stroke. 2008;39:2531-7.

30. Howlett JA, Northington FJ, Gilmore MM, Tekes A, Huisman TAGM, Parkinson C, et al. Cerebrovascular autoregulation and neurologic injury in neonatal hypoxic-ischemic encephalopathy. Pediatr Res. 2013;74:525-35.

31. Lee JK, Poretti A, Perin J, Huisman TAGM, Parkinson C, Chavez-Valdez R, et al. Optimizing cerebral autoregulation may decrease neonatal regional hypoxic-ischemic brain injury. Dev Neurosci. 2017;39:248-56.

32. Lee JK, Brady KM, Chung S-E, Jennings JM, Whitaker EE, Aganga D, et al. A pilot study of cerebrovascular reactivity autoregulation after pediatric cardiac arrest. Resuscitation. 2014;85:1387-93.

33. Burton VJ, Gerner G, Cristofalo E, Chung S, Jennings JM, Parkinson C, et al. A pilot cohort study of cerebral autoregulation and 2-year neurodevelopmental outcomes in neonates with hypoxic-ischemic encephalopathy who received therapeutic hypothermia. BMC Neurol. 2015;15:209.

34. Pham P, Bindra J, Chuan A, Jaeger M, Aneman A. Are changes in cerebrovascular autoregulation following cardiac arrest associated with neurological outcome? Results of a pilot study. Resuscitation. 2015;96:192-8.

35. Lavinio A, Timofeev I, Nortje J, Outtrim J, Smielewski P, Gupta A, et al. Cerebrovascular reactivity during hypothermia and rewarming. $\mathrm{Br} J$ Anaesth. 2007;99:237-44

36. Steiner L a, Czosnyka M, Piechnik SK, Smielewski P, Chatfield D, Menon DK, et al. Continuous monitoring of cerebrovascular pressure reactivity allows determination of optimal cerebral perfusion pressure in patients with traumatic brain injury. Crit Care Med. 2002;30:733-8.

37. Jaeger M, Schuhmann MU, Soehle M, Meixensberger J. Continuous assessment of cerebrovascular autoregulation after traumatic brain injury using brain tissue oxygen pressure reactivity. Crit Care Med. 2006;34:1783-8.

38. Aries MJ, Czosnyka M, Budohoski KP, Steiner LA, Lavinio A, Kolias AG, et al. Continuous determination of optimal cerebral perfusion pressure in traumatic brain injury. Crit Care Med. 2012:40:2456-63.

39. Ghosh A, Elwell C, Smith M. Review article: cerebral near-infrared spectroscopy in adults: a work in progress. Anesth Analg. 2012;115:1373-83. 